\title{
FORMAS BULBARES DA SINDROME DE GUILLAIN-BARRÉ
}

\author{
Paulo Pinto Pupo* \\ Eliova Zukerman ** \\ Nelson Alvares Cruz ***
}

\author{
J. Geraldo de Camargo Lima **** \\ CEME JORDY $* * * *$ \\ Fernando Menezes Braga $* * * * *$
}

O acometimento dos nervos bulbares na síndrome de Guillain-Barré parecia-nos raro. Entretanto, nestes últimos 18 meses tivemos a oportunidade de observar 6 pacientes em que tal aconteceu, o que nos chamou a atenção para o fato e nos levou a empreender uma revisão do assunto. Nesta revisão deparamos com idéias por vêzes as mais desencontradas, quer em relação ao diagnóstico clíntico, quer no que diz respeito às formas clínicas, quer quanto à própria conceituação da síndrome individualizada por Guillain e Barré, quer em relação aos achados anátomo-patológicos.

Predomina hoje, entretanto, o conceito de que a afeç̧ão descrita por Guillain-Barré-Strohl, com um quadro clínico definido, de evolução favorável é, em última análise, conseqüência de uma reação alérgica ao nivel das raizes, nervos e mesmo do neuro-eixo, em face de fatôres desencadeantes os mais variáveis. Nesse conceito é que nós nos situamos. É à luz dêsses conhecimentos que focalizamos os nossos casos e é nêles que baseamos nosso diagnóstico.

É interessante enunciar êste ponto de vista, porque muitos casos de perturbações neuríticas de moléstias tóxicas ou infecciosas têm sido assinalados, em particular casos de afecçōes neuríticas relacionadas à difteria. $\mathrm{Na}$ literatura alguns dos casos de forma bulbar da polirradiculoneurite, o foram, como o de Gareiso ${ }^{31}$, e, em nosso próprio material clínico dois casos foram tidos como tendo sofrido difteria anteriormente, e um dêles realmente sofreu desta afecção (caso 7). Seriam êles realmente neurites tóxicas, ou seriam verdadeiras polirradiculoneurites de natureza alérgica, desencadeadas pelo próprio estado infeccioso e evoluindo de modo benigno, tal como a sindrome de Guillain-Barré, acompanhando-se de dissociação albumino-citológica no LCR?

A eventualidade do comprometimento dos nervos cranianos nos casos de polirradiculoneurites generalizadas ou mesmo, a do comprometimento exclusivo dêsses nervos cranianos levou os diversos autores que têm tratado do assunto, a discutir classificações as mais variadas para formas clínicas

Trabalho do Serviço de Neurologia da Escola Paulista de Medicina (Prof. Paulino W. Longo. *Chefe de Clínica; ** Assistente; ***Assistente extranumerário do Serviço de Otorrinolaringologia (Prof. Paulo Mangabeira Albernaz); **** Médico interno; ***** Doutorando interno. 
diferentes da mesma afecção, relacionando a isto também o aspecto de maior ou menor gravidade dos casos clínicos de per si 1, 14, 16, 18, 38, 43, 62, 66, 69, $70,73,82,84,102,103,104,105$.

Naturalmente não interessa reviver essas discussões. Achamos mais interessante, a fim de situar na literatura os nossos casos, fazer uma análise da incidência do comprometimento dos nervos cranianos em grande número de casos, cuja descrição clínica encontramos bem discriminada na literatura ${ }^{1}$, $2,4,6,8,10,12,16,17,18,19,21,23,26,27,29,31,33,34,39,40,43,44,47,48,53,54,55$, $56,58,59,63,64,65,68,71,73,74,77,78,79,83,84,85,86,88,93,94,95,97,98,99,101,102$, $103,104,105,106$. Com êles elaboramos o gráfico 1 , referente a 189 pacientes diferentes, estando projetados quantas vêzes cada nervo craniano foi comprometido.

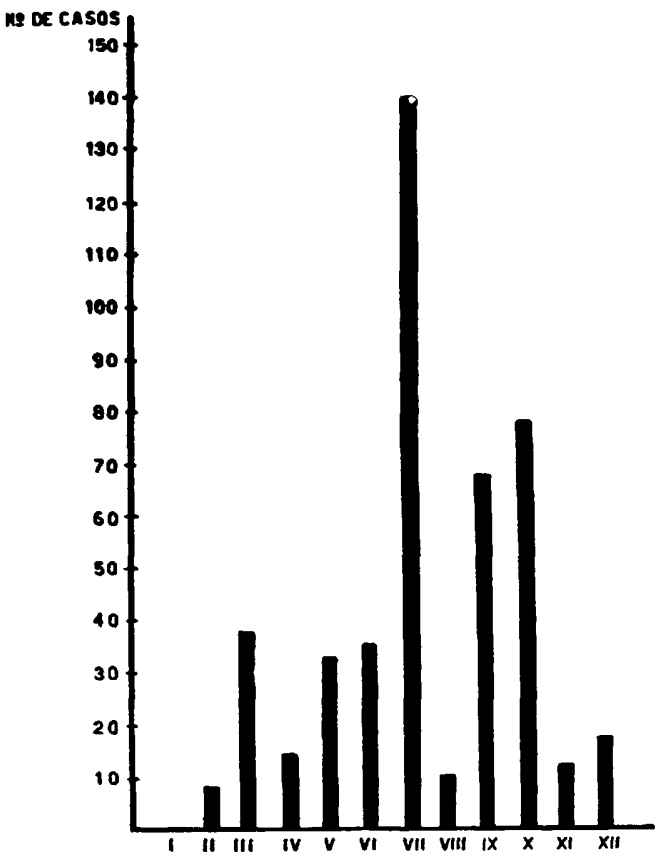

Gráfico 1 - Freqüência de acometimento de cada nervo craniano: análise de 189 casos da literatura.

Da análise dêsse quadro ressalta, logo, a predominância do acometimento do nervo facial, lesado em 140 de 189 casos. A seguir se situam os nervos bulbares, glossofaríngeo e pneumogástrico, lesados respectivamente em 69 e 78 casos. Os nervos espinal e hipoglosso foram acometidos em proporção muito menor nessa casuística (11 e 18 vêzes respectivamente). 
Dos demais nervos cranianos acometidos assinalam-se, em ordem decrescente de incidência, os nervos motor ocular comum, motor ocular externo, trigêmeo, patético, acústico e óptico. O nervo olfativo não foi citado como tendo sido acometido.

O comprometimento dos nervos bulbares não é, pois, uma raridade quando associadamente a outros nervos cranianos, em particular ao nervo facial. Entretanto, muito menos freqüente é o acometimento isolado dêsses nervos. No gráfico 2, isto é bem evidente, pois em apenas 21 dos 189 casos êsses foram os únicos nervos cranianos acometidos, mas, assim mesmo, associados ao acometimento dos membros. Em um caso apenas o seu acometimento foi exclusivo.

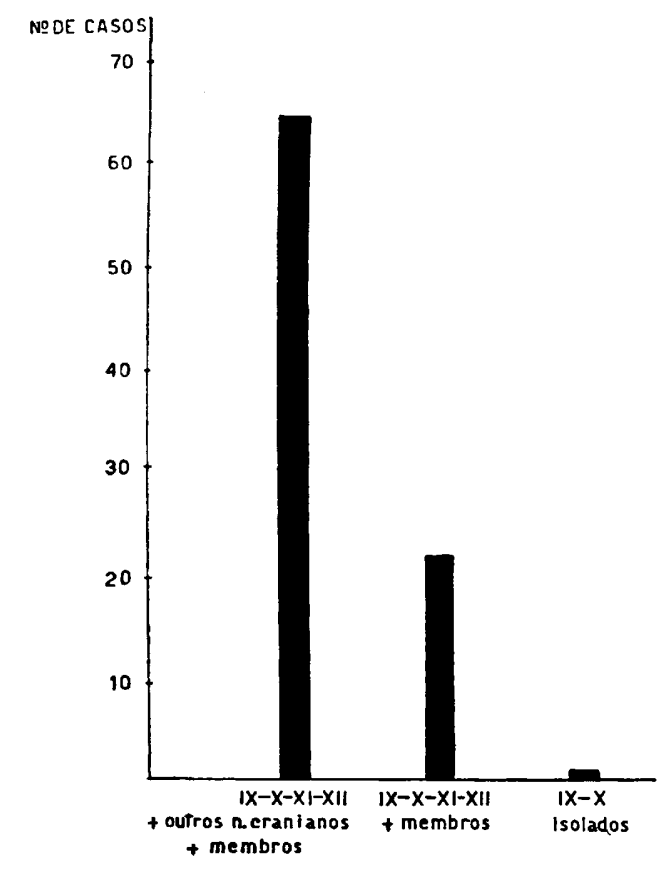

Gráfico 2 - Freqüência de acometimento dos nervos bulbares: análise de 189 casos da literatura.

No Serviço de Neurologia da Escola Paulista de Medicina, de 1939 a 1959 foram registrados 18 casos de síndrome de Guillain-Barré, seguidos em tempo suficiente para que êsse diagnóstico pudesse ser bem afirmado. Dêsses 18 pacientes, 9 apresentavam sòmente paralisia dos membros; um apresentava, além da paralisia dos membros, o acometimento do nervo facial bilateralmente; em um havia paralisia dos membros e do nervo motor ocular externo; em 7 havia comprometimento dos nervos bulbares. É esta última 
eventualidade que se destaca pela alta incidência de comprometimento dos nervos bulbares e pelo fato de a maioria dos casos ter aparecido em tempo relativamente curto, o motivo do presente trabalho. Vamos analisar, pois, cada um dos 7 casos de sindrome de Guillain-Barré em que o acometimento dos nervos bulbares estêve presente.

\section{OBSERVACOES}

CAso 1 - E.R.P., 5 anos, sexo feminino, branca, brasileira, matriculada em 14-1958. Há dois meses, febre e dor nas regiões parotidianas, seguindo-se entumescimento retromandibular bilateral; feito tratamento com antibióticos e cortisona, regrediram completamente, em poucos dias, os sintomas da parotidite. Duas semanas após começou a apresentar dificuldade na deglutição e voz rouca, que se tornou, depois, anasalada; a seguir, instalou-se progressivamente fraqueza geral e dôres nos braços e pernas. O quadro sintomático foi piorando progressivamente. Exame geral - Paciente abatida, emagrecida e afebril; pressão arterial $110 \times 70$. Exame neurológico - Abolição dos reflexos profundos dos membros superiores e inferiores, nos quais não se notava qualquer alteração do tonus ou da motilidade voluntária. Paralisia do véu do paladar. Ausência dos reflexos faringeo e de náusea, assim como dificuldade acentuada na deglutição, com refluxo de alimentos pelo nariz; voz anasalada; paresia da corda vocal esquerda. Exame de secreção amigdaliana - negativo para bacilo diftérico. Exame do líqüido cefalorraqueano lombar - liquor límpido e incolor, formação de reticulo fibrinoso; 1,8 células por $\mathrm{mm}^{3} ; 98 \mathrm{mg} \%$ de proteinas totais; opalescência às reações de Nonne, Weichbrodt e Pandy; reação de Takata-Ara positiva, tipo floculante; $731 \mathrm{mg} \%$ de cloretos; $63 \mathrm{mg} \%$ de glicose; reação do benjaim coloidal 00000.22222.20000.0; reações de Wassermann, de Steinfeld, de Eagle e do desvio do complemento para cisticercos, negativas; em sintese, dissociação albumino-citológica. Diagnóstico: Sindrome de Guillain-Barré. Tratamento: vitamina $\mathrm{B}_{1}$, tetraciclina, sulfato de estriquinina e predinisolona. Evolucūo Em 5 de agôsto de 1958, reflexos profundos normais, com excecão dos aquilianos que persistem abolidos; motilidade normal do véu do paladar; reflexos faríngeo e de náusea, normais; deglutição normal; voz normal. Exame do LCR lombar liquor limpido e incolor; 1,8 células por $\mathrm{mm}^{3} ; 32 \mathrm{mg} \%$ de proteinas totais; reaçōes de Pandy, Weichbrodt e Nonne negativas; reação do benjoim coloidal 00000.02220.00000.0; reação de Takata-Ara negativa; demais reações normais; em síntese, taxa de proteínas no limite superior de normalidade. O caso evoluiu para a cura em 4 meses.

Sintese - Quadro cínico de inicio infeccioso geral, arreflexia profunda generalizada, dôres radiculares e comprometimento dos IX e X nervos cranianos; evolução favorável, cura em 4 meses, tendo como seqüela arreflexia aquiliana.

CASo 2 - A.C.L.T., 7 anos, sexo feminino, branca, brasileira, matriculada em 16-4-1958. Há 3 meses, estado aparentemente gripal, com dôres no corpo, abatimento geral e febre; logo no início surgiu dificuldade na deglutição tanto para sólidos como para líqüidos, com refluxo pelo nariz; a voz inicialmente rouca, tornou-se ulteriormente imperceptivel, indo até à afonia. Vinte dias após, apareceram dôres na região cervical, sendo a cabeça mantida permanentemente inclinada para a direita. O médico assistente fêz tratamento com antibióticos e vitaminas, sem resultado. O quadro clínico foi piorando progressivamente até a data atual. Exame geral - Paciente abatida; pulso 80 ; pressão arterial $80 \times 60$; temperatura $36,5^{\circ} \mathrm{C}$. Exame neurológico - Inclinação da cabeça para a direita, hipotonia do músculo externocleidomastoideu e trapézio à esquerda; paresia das cordas vocais. Nos mem- 
bros superiores e nos inferiores não havia alteração da motilidade, dos reflexos ou do tonus. Dóres radiculares cervicais bilaterais, mais intensas à direita. Exame radiográfico simples do crânio, normal. Exame radiográfico simples da coluna cervical: escolíose sinistro-convexa da coluna cervical; transição crânio-vertebral normal. Exame para a pesquisa do bacilo diftérico na secreçió faringea, negativo. Exame do líüido cefalorraqueano lombar - liquor limpido e incolor; 1,8 células por $\mathrm{mm}^{3} ; 84 \mathrm{mg} \%$ de proteinas totais; opalescência às reações de Nonne e Pandy; $720 \mathrm{mg} \%$ de cloretos; $54 \mathrm{mg} \%$ de glicose; reação do benjoim coloidal 00000.12222. 20000.0; reações de Wassermann, de Steinfeld, de Eagle, negativas; em sintese, dissociação albumino-citológica. Diagnóstico: Sindrome de Guillain-Barré. Tratamento: Corticóides, sulfato de estriquinina e vitamina $\mathrm{B}_{\mathfrak{1}}$. Evoluçăo $-\mathrm{Em} 26$ de abril de 1958, regressão completa de todo o quadro clínico; a paciente falava bem, a deglutição já era normal e a cabeça assumia a sua posição normal; a fôrça muscular e o tonus dos músculos externocleidomastoideu e trapézio estavam normais.

Sintese - Caso iniciado por processo infeccioso geral, com dôres radiculares cervicais; comprometimento dos IX e X nervos cranianos, bilateralmente, e do XI à direita; evolução favorável em 10 dias.

Caso 3 - M.R.L., 9 anos, sexo feminino, branca, brasileira, matriculada em 304-1958. Há dois meses estado aparentemente gripal, com cefaléia, dôres no corpo, abatimento geral, febre e dor de garganta. Quatro dias após, dificuldade de deglutição, tanto para sólidos como para líquiidos que refluiam pelo nariz; voz anasalada. Há 15 dias, visão dupla e cefaléia moderada. Exame geral - Paciente abatida; pulso 80 ; pressão arterial $110 \times 70$; temperatura $36^{\circ} \mathrm{C}$. Exame neurológico - Globos oculares desviados para dentro, com impossibilidade de excursão lateral; voz anasalada; paresia bilateral do véu do paladar; ausência dos reflexos faringeo e de náusea; incapacidade de deglutição voluntária, com refluxo de alimentos pelo nariz; paresia das cordas vocais. Não havia distúrbios da sensibilidade. Exame do líqüido cefalorraqueano suboccipital - Aspecto limpido e incolor; 1 célula por $\mathrm{mm}^{3} ; 32 \mathrm{mg} \%$ de proteinas totais; opalescência à reação de Pandy; $725 \mathrm{mg} \%$ de cloretos; $58 \mathrm{mg} \%$ de glicose; reação do benjoim coloidal 00000.02220.00000.0; reações de Takata-Ara, Wassermann, Steinfeld e Eagle e do desvio do complemento para cisticercos, negativas; em sintese, dissociação albumino-citológica. Diagnóstico: Sindrome de Guillain-Barré. Tratamento: Sulfato de estriquinina, vitamina $B_{1}$ e prednisolona. Evolução - Em 14 de maio de 1958, regressão total das paresias oculares e dos distúrbios da deglutição e da fonação, com motilidade normal do véu do paladar e dás cordas vocais; reflexos faríngeo e de náusea normais.

Sintese - Caso de início infeccioso geral com comprometimento bilateral dos VI, IX e X nervos cranianos; evolução para cura após 16 dias.

Caso 4 - A.M.F., 7 anos, sexo masculino, branco, brasileiro, matriculado em 10-6-1958. Há dois meses. fehre e dor de garganta; o médico assistente administrou penicilina, com o que os sintomas desapareceram completamente. Um mês após, o paciente foi amigdalectomizado; no terceiro dia de pós-operatório o paciente já se apresentava normal retornando à escola; no quinto dia começou a se queixar de dor de cabeça e, no sexto dia, o paciente apresentava-se enfraquecido, mal podendo caminhar, com a voz anasalada e a deglutição dificultada, inclusive para liqüidos, os quais refluiam pelo nariz. O quadro clinico foi piorando progressivamente até a data atual. Exame geral - Paciente abatido, afebril; pressão arterial $90 \times 60$. Exame neurológico - Paraparesia flácida, com abolição dos reflexos patelares, aquilianos e médio-plantares; reflexos cutâneo-plantares normais. Nos membros superiores diminuição dos reflexos profundos e da fôrça muscular, sem paresia nitida. Não havia distúrbios da sensibilidade. Paresia do véu do pa- 
ladar; ausência do reflexo faringeo e do reflexo de náusea; dificuldade acentuada para a deglutição; voz anasalada; refluxo de alimentos pelo nariz. Exame da secrešio amigdaliana: negativo, para bacilo diftérico. Exame do líqüido cefalorraqueano lombar: liquor limpido e incolor; 1 célula por $\mathrm{mm}^{3} ; 64 \mathrm{mg} \%$ de proteinas totais; opalescência às reações de Nonne e Pandy; $749 \mathrm{mg} \%$ de cloretos; $65 \mathrm{mg} \%$ de glicose; reação do benjoim coloidal 00000.22222 .00000 .0 ; reaçōes de Wassermann, do desvio do complemento para cisticercos, de Steinfeld, de Eagle, negativas; em síntese, dissociação albumino-citológica. Diagnóstico: Sindrome de Guillain-Barré. Tratamento: Prednisolona, sulfato de estriquinina, vitamina $B_{t}$ e vitamina C. Evolucão - Em 24 de junho de 1958, melhora acentuada da motilidade dos membros inferiores, já com possibilidade de se manter bem de pé, marcha pràticamente normal; reflexos patelares, aquilianos e médio-plantares presentes, mas hipoativos; nos membros superiores, os reflexos estavam normais, não havendo mais diminuição da fôrça muscular; reflexo faringeo presente, deglutição normal, voz ainda um pouco anasalada.

Sintese - Quadro com início infeccioso, paresia flácida dos membros inferiores, comprometimento dos IX e $\mathrm{X}$ nervos cranianos; evolução favorável para a cura em 14 dias.

CASo 5 - W.C.L., 4 anos, sexo masculino, branco, brasileiro, matriculado em 3-6-1958. Há 45 dias, febre e dor de garganta. O médico assistente suspeitou de difteria; entretanto os exames subsidiários não confirmaram essa hipótese; foi instituido tratamento com antibióticos e sôro anti-diftérico, não ocorrendo melhoras apreciáveis. Há 25 dias, fraqueza nos membros inferiores, dificuldade de deglutição e alterações da voz. O quadro clínico foi piorando progressivamente até a data atual. Exame geral - Paciente abatido, pálido; temperatura $36,5^{\circ} \mathrm{C}$, axilar; pulso 100 por minuto. Exame neurológico - Paraplegia flácỉda com abolição dos reflexos patelares, aquilianos e médio-plantares; reflexos cutâneo-plantares normais. Nos membros superiores a motilidade, o tonus e os reflexos profundos estavam normais. Hiperestesia táctil e dor à compressão muscular nos membros inferiores. Paresia bilateral do véu do paladar. Ausência do reflexo faríngeo e de náusea; incapacidade para deglutição voluntária; voz anasalada; refluxo de alimentos pelo nariz. Não havia nitida alteração da motilidade das cordas vocais. Incapacidade de excursão do ôlho esquerdo para fora. Exame do liqüido cefalorraqueano lombar - Liquor limpido e incolor; 0,4 células por $\mathrm{mm}^{3} ; 180 \mathrm{mg} \%$ de proteinas totais; opalescência às reações de Pandy e Nonne; $725 \mathrm{mg} \%$ de cloretos; $64 \mathrm{mg} \%$ de glicose; reaçôes de Wassermann, do desvio do complemento para cisticercos, de Steinfeld, negativas; reação do benjoim coloidal 02220.22222.22222.0; em síntese, dissociação albumino-citológica. Diagnóstico: Síndrome de Guillain-Barré. Tratanento: ACTH, vitamina $B_{1}$ e sulfato de estriquinina. Evoluçio - Melhora progressiva do quadro clínico, tendo o paciente recebido alta hospitalar 10 dias após, apresentando ainda paresia flácida dos membros inferiores, abolição dos reflexos patelares, aquilianos e médio-plantares; marcha possivel com apoio. Persistia o distürbio da fonação, porém não mais a dificuldade de deglutição, nem o refluxo de líqüidos pelı) nariz. Exame do líqüido cefalorraqueano: Líquor límpido e incolor; 1 célula por $\mathrm{mm}^{3} ; 195 \mathrm{mg} \%$ de proteínas totais; opalescência às reações de Pandy e Nonne; $731 \mathrm{mg} \%$ de cloretos; $62 \mathrm{mg} \%$ de glicose; reação de Wassermann negativa; reação do benjoim coloidal 00000.20000.00000.0. Foi receitado prednisolona, vitamina $B_{1} e$ sulfato de estriquinina. Reexaminado a 5 de agôsto de 1958, o paciente apresentava marcha com pequena instabilidade, fôrça e tonus muscular nos membros inferiores normais; reflexo patelar hipoativo à direita, normal à esquerda; reflexos aquilianos ausentes bilateralmente; voz pouco anasalada; paresia do pálato mole à direita pouco evidente; reflexos faríngeo e de deglutiçâo normais; motilidade ocular 
normal. Novo exame do líqüido cefalorraqueano: Liquor límpido e incolor; 1,4 células por $\mathrm{mm}^{3} ; 60 \mathrm{mg} \%$ de proteinas totais, opalescência às reações de Pandy e Nonne. Em sintese, dissociação albumino-citológica menos acentuada.

Síntese - Quadro clínico que se iniciou com distúrbios da deglutição e da fonação, seguidos, 20 dias após, progressivamente, de paraplegia flácida. O exame neurológico revelou paralisia dos VI, IX e $\mathrm{X}$ nervos cranianos, associada a paralisia periférica dos membros inferiores, com dor à compressão muscular e hiperestesia táctil. Melhora em 15 dias, com alta hospitalar; cura em 60 dias, com sequielas minimas.

CAso 6 - I.P., branca, 24 anos, brasileira, casada, examinada em 2-3-1955. Há 3 semanas parestesias nas extremidades distais dos quatro membros; há 8 dias, díficuldade na movimentação dos membros inferiores, há 4 dias, déficit funcional dos membros superiores, assim como impossibilidade para levantar a cabeça do travesseiro; nesta ocasião começou a ter diplopia e dificuldade para deglutir alimentos sólidos e liqüidos. Exame geral - Paciente abatida e afebril. Exame neurológico Tetraparesia intensa, restando apenas alguns movimentos globais dos membros superiores e dos dedos das mãos; arreflexia profunda generalizada. Paresia facial de tipo periférico de moderada intensidade; paralisia total e bilateral dos III, IV e VI nervos cranianos, com impossibilidade de movimentação dos globos oculares em tôdas as direçōes; paresia dos $\mathrm{IX}$ e $\mathrm{X}$ nervos cranianos com grande dificuldade na deglutição e abolição dos reflexos faringeo e de náusea; paresia dos músculos trapézio e externocleidomastóideo (XI nervo craniano), bilateralmente. Exame do liqüido cefalorraqueano lombar - Líquor xantocrômico; $380 \mathrm{mg} \%$ de proteinas; 1 célula por $\mathrm{mm}^{3}$; formação de retículo fibrinoso; reações para globulinas positivas; $714 \mathrm{mg} \%$ de cloretos; $58 \mathrm{mg} \%$ de glicose; reação do benjoim coloidal 22222.22000 . 22222.0; reação de Takata-Ara positiva; tipo vermelho; reações de Wassermann, Steinfeld, Eagle e do desvio do complemento para cisticercos, negativas; em sintese, dissociação albumino-citológica. Diagnóstico: Sindrome de Guillain-Barré. Tratamento: ACTH endovenoso, salicilato de sódio endovenoso e estriquinina. Evoluçao - Após 3 a 4 dias de tratamento, a paciente já conseguia deglutir bem, desaparecendo progressivamente as paralisias oculares, tendo alta hospitalar após 38 dias de internação. Nesta ocasião havia apenas fraqueza muscular generalizada, embora conseguisse andar perfeitamente. Restava ainda abolição dos reflexos profundos. Novo exame do líqüido cefalorruqueano, feito 20 dias após o primeiro, mostrou dissociação albumino-citológica ( $820 \mathrm{mg} \%$ de proteínas e 0,4 células por $\mathrm{mm}^{3}$ ); reações para globulinas positivas; demais elementos normais. Reexaminada 26 dias após alta hospitalar, a paciente apresentava-se perfeitamente bem, sem qualquer alteração, restando apenas arreflexia profunda generalizada.

Sintese - História de três semanas; quadro clinico de parestesias, depois tetraparesia flácida com arreflexia profunda generalizada e comprometimento dos III, VI, VII, IX, X e XI nervos cranianos; evolução favorável e cura em dois meses, restando apenas arreflexia profunda generalizada.

Caso 7 - E.A.C., 4 anos, sexo feminino, branca, brasileira, examinada em 9-51959. Há um mês a paciente estêve internada no Hospital de Isolamento por ter difteria; permaneceu 8 dias em tratamento saindo perfeitamente bem, sem qualquer seqüela ou distúrbio objetivo. Após 8 dias, estrabismo e dificuldade para apreender objetos, andar, abaixar e levantar. Estes sintomas progrediram, apresentando também dificuldade de deglutição e fonação, não conseguindo engolir liqüidos ou sólidos e só emitindo alguns sons. Exame geral - Pacjente abatida, afebril. Exame neurológico - Déficit do movimento lateral para fora dos globos oculares, bilateralmente, mais à direita; paresia do véu do paladar com dificuldade para degluti- 
ção; abolição dos reflexos faríngeo e de náusea; não havia alteração nitida da movimentação das cordas vocais; paresia acentuada nos quatro membros, com maior déficit muscular nos músculos das raizes dos membros; arreflexia profunda generalizada. Exame do liqüido cefalorraqueano lombar - Liquor limpido e incolor; 0,8 células por $\mathrm{mm}^{3} ; 82 \mathrm{mg} \%$ de proteinas totais; opalescência às reações para globulinas; $708 \mathrm{mg} \%$ de cloretos, $48 \mathrm{mg} \%$ de glicose, reação do benjoim coloidal 22222.22200.02222.0; reação de Takata-Ara positiva, tipo vermelho; reações de Wassermann, Eagle, Steinfeld e desvio de complemento para cisticercos, negativas; em sintese, dissociação albumino-citológica. Diagnóstico: Síndrome de Guillain-Barré. Tratamento: Dexametazona, estriquinina, vitamina $\mathrm{B}_{1}$ e complexo B. Evolucāo A paciente melhorou progressivamente e, após um mês, apresentava-se perfeitamente bem do ponto de vista geral e neurológico, com ótima movimentação geral, regredindo todos os sinais de comprometimento de nervos cranianos e os distúrbios motores dos membros, restando apenas arreflexia profunda generalizada.

Síntese - Quadro de polirradiculoneurite, instalado 8 dias após cura clínica de difteria, com comprometimento bilateral dos VI, IX e $\mathrm{X}$ nervos cranianos, tetraparesia flácida com arreflexia profunda generalizada. Líquor com dissociação albumino-citológica. Evolução para cura em 30 dias.

\section{COMENTARIOS}

O comprometimento dos nervos bulbares IX e X (glossofaríngeo e pneumogástrico) foi comum a todos os casos aqui registrados. Acometimento menos freqüente foi o do XI nervo (espinal), evidente sòmente nos casos 2 e 6. O XII nervo (hipoglosso) em caso algum foi lesado.

A afecção dêsses nervos bulbares se apresentou isolada sòmente nos casos 2 e 3. Nos demais casos ela se apresentou associada à afecção de membros ( $\operatorname{casos} 1,4,5,6$ e 7) ou à do nervo abducente (casos 3, 5, 6 e 7), do nervo motor ocular comum (caso 6), do nervo facial (caso 6). Chama a atenção o fato de o comprometimento do nervo facial ter se apresentado sòmente no caso 6 , em contraste com o que se vê na literatura, onde êste nervo craniano é considerado como o mais freqüentemente afetado.

Como comentário final resta assinalar o fato de que a lesão dos nervos bulbares na síndrome de Guillain-Barré não foi eventualidade rara em nossa casuística.

\section{RESUMO}

Uma revisão crítica do conceito, das formas clínicas, das teorias patogênicas relativas à síndrome de Guillain-Barré é feita preliminarmente, com finalidade de situar o ponto de vista dos autores em relação às idéias que encontraram na literatura, por vêzes bastante divergentes. A seguir, uma revisão relativa ao comprometimento dos nervos cranianos nos casos publicados veio mostrar que o nervo facial é o mais freqüentemente atingido, sendo bastante mais raro o comprometimento dos nervos motores oculares ou dos nervos bulbares. 
São relatadas 7 observaçōes de casos de sindrome de Guillain-Barré com dissociação albumino-citológica no líqüido cefalorraqueano e evolução favorável para a cura em tempo relativamente curto, nos quais a lesão dos IX e $\mathrm{X}$ nervos cranianos foi constante. Este acometimento foi isolado em 2 casos (em um dêles tendo sido também lesado o XI nervo), associada a outros nervos cranianos não bulbares em 4 casos e à paralisia de membros em 5 pacientes.

Particular atenção merece o fato de estarem estas 7 observações incluidas em uma casuística de 18 casos de síndrome de Guillain-Barré, 6 dos quais observados no Serviço de Neurologia da Escola Paulista de Medicina em um curto periodo de 18 meses. Sua alta incidência contrasta, pois, com o que é habitualmente assinalado na literatura.

\section{SUMMARY}

The bulbar forms of the Guillain-Barré syndrome.

The ideas found in the medical literature about clinical and pathological aspects concerning the Guillain-Barré syndrome are varied; the authors try in this paper to give their opinion on the subject.

As far as the cranial nerves are concerned, the literature shows that the VIIth is doubtless the more frequently injured; the oculomotor and bulbar nerves are seldom damaged.

This study was done in 7 patients selected from 18 cases with GuillainBarré syndrome in the Serviço de Neurologia da Escola Paulista de Medicina. All 7 presented an albumino-cytologic dissociation in the C.S.F., a swift recovery towards cure and envolvement of the $\mathrm{IX}^{\mathrm{th}}$ and $\mathrm{X}^{\text {th }}$ nerves. One case presented isolated lesion of the $I^{\text {th }}$ and $X^{\text {th }}$ nerves; one case, involvement of the $\mathrm{IX}^{\mathrm{th}}, \mathrm{X}^{\mathrm{th}}$ and $\mathrm{XI}^{\text {th }}$ nerves; four cases, associated lesion of the $\mathrm{IX}^{\mathrm{th}}, \mathrm{X}^{\mathrm{th}}$ and others than bulbar nerves; four cases, paralysis of the limbs besides involvement of the cranial nerves.

The authors call attention to high incidence of the bulbar form of the Guillain-Barre syndrome in their material, in contrast with what has been reported in the literature ( 7 bulbar cases out of 18 Guillain-Barré syndrome patients).

\section{REFERENCIAS}

1. ALAJOUANINE, Th.; DELAY, J.; THUREL, R.; HORNET, Th.; BOUDIN, G. - The polyradiculonévrite aigüe genéralisée avec diplégie faciale et paralisie terminale des muscles respiratoires et avec dissociation albumino-cytologique. Etude natomique. Rev. Neurol., 65:681-697, 1936. 2. ALAJOUANINE, Th.; HORNET, Th.; BOUDIN, G. - Etude anatomo-clinique d'un cas de polyradiculonévrite aigüe generalisée avec diplégie faciale et dissociation albumino-cytologique et mort au $8 \mathrm{e}$. jour par paralysie respiratoire. Rev. Neurol., 73:547-562, 1941. 3. ALPERS, B. J. Clinical Neurology, 2a edição. F. A. Davis Co., Filadélfia, 1952, págs. 157-159. 4. 
ARING, C. D.; SABIN, A. B. - Fatal infectious polyneuritis in childhood. Arch. Neurol. a. Psychiat., 47:938-942, 1942. 5. BARRE, J. A. - Considerations sur le syndrome de polyradiculonévrite avec dissociation albumino-cytologique. J. Belge de Neurol. et Psychiat., 38:313-317, 1938. 6. BENDZ, P. - Respiratory problems in acute Guillain-Barré syndrome. Arch. Neurol. a. Psychiat., 73:22-27, 1955. 7. BIEMOND, A. - Quelques remarques sur l'etiologie de la maladie de Guillain-Barré. J. Belge de Neurol. et Psychiat., 38:231-239, 1938. 8. BLOOD, A.; LOCHE, W.; CARABASI, R. - Guillain-Barré syndrome treated with corticotropin (ACTH). J.A.M.A., 152:139-140, 1953. 9. BOSHES, B.; SHERMANN, I. C. - Variability of the course of Guillain-Barré syndrome. Neurology, 3:335, 1953. 10. BRADFORD, J.R.; BASFORD, E. F.; WILSON, J. A. - Acute infective polyneuritis. Quart. J. Med., 12:88-126, 1918-1919. 11. BRAIN, R. - Diseases of the Nervous System, $4^{\text {a }}$ edição. Oxford University Press. Londres, 1951, págs. 814-819. 12. BRISKIER, A. A. - Unusual rapid evolution in Guillain-Barré syndrome with bulbar palsy. J. Nerv. a. Ment. Dis., 100:462-465, 1944. 13. BROGGI, E.; BARBERA, G. - Cit. in Spiegel - Progress in Neurol, a. Psychiat., 9:174, 1954. 14. BROWN, J. R.; BAKER, A. B. - Diagnosis of GuillainBarré's disease. Am. J. Med,, 2:45-52, 1947. 15. CHUZID, J. G.; MARQUARDT, G. H. - Onset of Guillain-Barré syndrome following exposure to mustard gas. Arch. Neurol. a. Psychiat., 55:57-58, 1946. 16. COIRAULT, R.; LARCAN, A.; DAVIDOU, P. - Le Syndrome de Guillain-Barré et les Palyradiculonévrites Aigües avec Dissociation Albumino-cytologique dans le Liquide Céphalo-rachidien. Masson et Cie., Paris, 1958. 17. CREATURO, N. E. - Infectious mononucleosis and polyneuritis (Guillain-Barré syndrome): report of a case of facial diplegia treated with 2,3 dimercaptopropanol (B.A.L.). J.A.M.A., 143:234-236, 1950. 18. DAGNELIE, J. - Remarques sur quelques observations de polyradiculonévrites avec dissociation albumino-cytologique et à évolution favorable. J. Belge de Neurol. et Psychiat., 38:282306, 1938. 19. DARDENNE, M. M.; CAIZERQUES; RIBAUT - Syndrome de Guillain-Barré a forme pseudo-myopathique. Rev. Oto-Neuro-Ophtal., 23:126, 1951.20. DEBRE, R.; THIEFFRY, S. - Remarques sur le syndrome de Guillain-Barré chez l'enfant (a propos de 32 observations personelles). Arch. Franç. de Pediat., 8:357364, 1951. 21. DEMPSEY, W. S.; KARNOSH, L. S.; GARDNER, J. W. - GuillainBarré syndrome. Review of literature. Cleveland Clin. Quart., 14:206-217, 1947.22. DRAGANESCO, S.; CLAUDIAN, J. - Sur un cas de radiculonévrite curable apparue au cours d'une ostéomiélite du bras. Rev. Neurol., 2:517-518, 1927. 23. DRAGANESCO, S.; FACON, E. - Polyradiculonévrites primitives en Roumanie. Paris Méd., 103:411-414, 1937. 24. DROUET, P. L.; FAZURE, G.; LAMY, P.; LARCAN, A. -Resumo in Excerpta Médica, seç̧̃o VIII, 1956. 25. DUMOLARD; LARROUY; SCHOSBOE; BADAROUX - Polyradiculonévrite avec hỷperalbuminose du liquide céphalorachidien sans réaction cellulaire et névrite optique. Évolution rapide vers la guérison (syndrome de Guillain-Barré). Rev. Oto-Neuro-Ophtal., 15:26-28, 1937.26. FISCHER, M. - An unusual variant of acute idiopathic polyneuritis (syndrome of ophthalmoplegia, ataxia and arreflexia). New England J. Med., 255:57-65, 1956.27. FRAGNITO, O. - Sulla polinevrite acuta febrile con participazione del simpatico cervicale. Riv. Neurol., 1:117-136, 1928. 28. FRANCO, F. - Sulla patogenesi allergica delle polinevriti sistematizzate. Cervello, 23:321-352, 1947. 29. FRANÇOIS, M. M.; ZUCCOLI, G.; MONTUS, G. - Sur un cás de polyradiculoneurite curable avec dissociation albumino-cytologique: syndrome de Guillain-Barré. Rev. Neurol., 1:9597, 1929. 30. FURTADO, D.; VASCONCELOS, A. - Sindrome de Guillain-Barré consecutivo a gripe. Arq. Neuro-Psiquiat., 7:6-21, 1949. 31. GAREISO, A.; SAGRERAS, P. O. - Poliradiculoneuromiositis con sindrome de Guillain-Barré de etiologia diftérica. Arch. Argent. Pediat., 2:418-430, 1938. 32. GARVEY, P. H.; JONES, N.; WARREN, S. L. - Polyradiculoneuritis (Guillain-Barré syndrome) following the use of sulfanilamide and fever therapy. J.A.M.A., 115:1955-1962, 1940. 33. GARVIN, J. S. - Infectious mononucleosis with Guillain-Barré syndrome. J.A.M.A., 151:293-294, 1953. 34. GIORDANO, G. B. - Contributo alla conoscenza della poliganglioradicolite maligna. Rass. Studi. Psichiat., 32:305, 1943. 35. GIUBILEO, M. - Resumo in Excerpta Médica, Secção VIII:131, 1956. 36. GRANT, H.; LEOPOLD, H. N. Guillain-Barré syndrome occurring during cortizone therapy. J.A.M.A., 155: 252-253, 
1954. 37. GRAZIANO, R. - La llamada polirradiculoneurites a forma pseudomiopatica: su discusión histopatológica. Rev. Argent. Neurol (Rosario), 11:154-167, 1946. 38. GUILlaiN, G. - Synthèse de la discussion sur les polyradiculonévrites (syndrome de Guillain-Barré). J. Belge de Neurol. et Psychiat., 38:323-329, 1938. 39. GUILlaIN, G. - Sur un cas de radiculonévrite avec hiperalbuminose du liquide céphalo-rachidien sans réaction cellulaire. Guérison complète, mais persistance de l'abolition des reflexes tendineux. Ses consequences pour les diagnostics d'avenir. Rev. Neurol., 65:799-801, 1936. 40. GUILLAIN, G. - Radiculoneuritis with acellular hyperalbuminosis of the cerebrospinal fluid. Arch. Neurol. a. Psychiat., 36:975-990, 1936. 41. GUILlaiN, G.; BARRE, J. A. - Quelques remarques sur notre syndrome de radiculonévrite avec hyperalbuminose du liquide céphalo-rachidien, sans réactions cellulaires. Rev. Neurol., 65:573-582, 1936. 42. GUILlaiN, G.; BARRE, J. A.; STROHL, A. - Sur un syndrome de radiculonévrite aves hyperalbuminose du liquide céphalo-rachidien sans réaction cellulaire. Remarques sur les caractères cliniques et graphiques des réflexes tendineux. Bull. Soc. Med. Paris, 40:462-470, 1916. 43. GUILLAIN, G.; KREIS, B. - Sur deux cas de polyradiculonévrite avec hyperalbuminose du liquide céphalo-rachidien sans réaction cellulaire. Paris Méd., 40: 244-247, 1937. 44. GUTIERREZ-MENDOZA, C. - Enfermedad de Guillain-Barré. Rev. de Neuro-Psiquiat. (Lima Peru), 9:294-318, 1946. 45. GUYTHER, J. R. - Cit. por Reitman e Rethschild 80. 46. HAGBERG, B.; HERLITZ, G. - Guillain. Barré syndrome associated with poliomyelitis in newborns. Acta Paediat., 42:179-190, 1953. 47. HAYMAKER, W.; KERNOHAN, J. - The Landry Guillain-Barré syndrome. Medicine, 28:59-141, 1949. 48. HELSMOORTEL, J.; MYLE, G. - Sur l'état des functions vestibulaires dans la polyradiculonévrite avec dissociation albumino-cytologique. Confin. Neurol., 4:147-158, 1942. 49. HOLMES, G. - Neurological complications in atypical pneumonia. Brit. Med. J., 4.492:218-220, 1947. 50. IVERSEN, F. - Acute polyradiculoneuritis arising after peritonsillar abscess accompanied by increased antiestreptolysin titer in cerebrospinal fluid. Acta Med. Scandinavica, 129:441-445, 1948. 51. JERVIS, G. A.; STRASSBURGER, P. J. - Guillain-Barré syndrome and acute anterior poliomyelitis. Am. J. Dis. Child., 65:431-439, 1943. 52. KANDEL, P. - Resumo in Excerpta Médica, seç̧ão VIII:390, 1956. 53. KLOVSTAD, O. - GuillainBarré's syndrome in infectious mononucleosis. Acta Med. Scandinavica, 138:67-70, 1950. 54. KRIZL, K. - Resumo in Excerpta Médica, secção VIII:896, 1956. 55. LAMARTINE DE ASSIS, J. - Sindrome de Guillain-Barré; a propósito de três casos, sendo um tratado pelo ACTH. Arq. Neuro-Psiquiat., 11:133-144, 1953. 56. LAMARTINE DE ASSIS, J.; SPINA FRANÇA NETO, A. - Sindrome de Guillain-Barré; a propósito de 5 casos tratados com ACTH e cortisona. Arq. Neuro-Psiquiat., 11:414419, 1953. 57. LEWEY, F. H. - What is the Guillain-Barré syndrome? J. Pediat., 26:165-177, 1945. 58. LOUIS-BAR, D.; PUISSANT, A.; DELREE, G. - Ophtalmoplegie bilaterale progressive avec atteinte de plusieurs autres nerfs crâniens et polyradiculite accompagnée de dissociation albumino-cytologique. J. Belge de Neurol. et Psychiat., 41-42:324-332, 1941-1942. 59. LOWENBERG, K.; FOSTER, D. B. - Polyradiculoneuritis with albumino-cytologic dissociation; pathologic report of 4 cases. Trans. Amer. Neurol. Ass., 70:98-102, 1944. 60. BARTA, L.; GERLOCZY, F. - Etudes des relations entre le syndrome de Guillain-Barré et les maladies a virus du système nerveux central. Arch. Franç. Pediat., 7:265-269, 1950. 61. MAFFEI, W. E. - As Bases Anátomo-patológicas da Neuriatria e Psiquiatria. Vol. 2. Imprensa Metodista, São Paulo, 1951, págs. 410-413. 62. MANCEAUX, A. G.; SUTTER, J. M.; PÉLICIER, Y. - Syndrome de Guillain-Barré à forme hyperalgique. Rev. OtoNeuro-Ophtal., 27:305, 1955. 63. McINTYRE, H. D.; KROUSE, H. - Guillain-Barré syndrome complicating antirabies inoculation. Arch. Neurol. a. Psychiat., 62:802. 808, 1949. 64. MARTIN, P.; TITECA, J. - Diplégie faciale curable. J. Belge de Neurol. et Psychiat., 38:217-227, 1938. 65. MASSION-VERNIORY, L. - Formes pseudo-diphteriques du syndrome polyradiculonévritique de Guillain-Barré. J. Belge de Neurol. et Psychiat., 40:306-309, 1940. 66. MASSION-VERNIORY, L. - Formes pseudo-myasthéniques à debut ophtalmoplégique du syndrome radiculonévritique de Guillain-Barré. J. Belge de Neurol. et Psychiat., 40:294-305, 1940. 67. MAZAUD, R.; GIUDICELli, P.; MOISSINAC, J. — L'épidemie de poliomyelite de 1954 à Dakar 
et les syndromes aberrants concomitants. Rev. Neurol., 92-93:195-216, 1955.68. MELLO, A. R. - Acèrca das polirradiculoneurites generalizadas com dissociação albumino-citológica. Brasil Méd., 53:951-954, 1939. 69. MICHON, P.; ALEXANDRE, G. - Les localisations hautes du syndrome de Guillain-Barré. Sem. Hôp. Paris, 12: 2939-2942, 1949. 70. MILLS, C. K. - The reclassification of some organic nervous diseases on the basis of the neurons. J.A.M.A., 31:11-13, 1898. 71. MONTADON, RAUCH, REYTAN - Mononucleosis Guillain-Barré syndrome and paralysis of the posterior crico-arytenoid muscle. Acta Otolaryngol., 46:35-42, 1956. 72. MORRISON, L. R. - Histopathologic effect of anoxia on the central nervous system. Arch. Neurol. a. Psychiat., 55:1-34, 1946. 73. MORSIER, G.; STEINMANN, J. - Les polyradiculonévrites: forme aigüe curable, forme à évolution fatale. Prèsse Méd., 94: 1890-1892, 1936. 74. NERI, O. - Das polirradiculonevrites (sindrome de GuillainBarré). Arq. Bras. Med., 34:351-367, 1944. 75. NERVEY, J. A.; LUBIN, R. I. Corticotropin (ACTH) therapy in Guillain-Barré syndrome. J.A.M.A., 1952:137-138, 1953. 76. NIELSEN, J. M. - Treatment of neuronitis with BAL. Bull. Los Angeles Neurol. Soc., 15:61-71, 1950. 77. PETERS, C. H.; WIDERMAN, A.; BLUMBERG, A.; RICKER, W. A. - Neurologic manifestations of infectious mononucleosis with special reference to the Guillain-Barré syndrome. Arch. Int. Med., 80:366-373, 1947. 78. PETSCHE, H. - Resumo in Excerpta Médica, secçāo VIII:774, 1953. 79. PINCKNEY, C. - Acute infective polyneurites with report of five cases. Brit. Med. J., 15:333-336, 1936. 80. REITMAN, N.; ROTHSCHILD, K. - The non-infectious nature of the Guillain-Barré syndrome with a possible explanation for the albumino-cytologic dissociation. Ann. Intern. Med., 32:923-934, 1950. 81. REYE, R. D. K. - Cit. in Year Book of Neurology, Psychiatry and Neurosurgery, pág. 189, 1954-1955. 82. RISER, M.; GERAUD, J.; GEIZES, L.; GARRIQUES, H. — Syndrome de GuillainBarré avec hypertension crânienne. Rev. Oto-Neuro-Ophtal., 28:122, 1956. 83. RISER, M.; GERAUD, J.; RASCOL; RISER, A.; SAINT MARC - Syndrome de Guillain-Barré-Strohl à évolution ascendante avec hypertension crânienne. Bull. Soc. Med. Paris, 73:689-693, 1957. 84. RISER, M.; PLANQUES, M. - Les polyradiculonévrites aigües (syndrome de Guillain-Barré et Strohl). J. Belge de Neurol. et Psychiat., 38:264-274, 1938. 85. ROGER, H.; BOUDOURESQUES, J. - Les polyradiculonévrites avec dissociation albumino-cytologique et à évolution favorable. J. Belge Neurol. et Psychiat., 38:243-255, 1938. 86. ROGER, H.; POURSINES, Y.; ROGER, J.; PUJOL, R. - Syndrome de Guillain-Barré avec paralisie des deux moteurs oculaires externes post-vaccination jennerienne. Rev. Oto-Neuro-Ophtal., 25:425-427, 1953.87. ROSEMAN, E.; ARING, C. D. - Infectious polyneuritis. Infectious neuronitis. Acute polyneuritis with facial diplegia, Guillain-Barré syndrome, Landry's paralysis. Medicine, 20:463-494, 1941. 88. ROUQUIS, L.; HILCEMAND, P.; GILBRIN, E. - Radiculonévrite à rechute; syndrome de Guillain-Barré probable. Rev. Neurol., 79:2032C5, 1947. 89. SCHEID, W.; WIECK, H. - Cit. in Spiegel - Progress Neurol. a. Psychiat., 6:120. 1951. 90. SCHEINKER, T. M. - Pathology and pathogenesis of infectious polynevritis. Guillain-Barré syndrome. J. Neuropathol. a. Exp. Neurol., 8:184-193, 1949. 91. SELTZER, H. S.; LICHTY, D. E.; CONN, J. W. - A case of Guillain-Barré syndrome (infectlous polyneuritis) with apparent response to ACTH. Univ. Michigan Med. Bull., 18:27-31, 1952. 92. SPINA-FRANCA, A. - Polineurites e polirradiculoneurites. Diagnóstico e tratamento. Rev. Med. (São Paulo), 35:248263, 1951. 93. SPOTA, B. B.; TAGLIABUE, C. A.; RIŨ, J. A. - Síndrome de Guillain-Barré-Strohl. Nova observação sôbre a forma pseudotábida encefálica. Arq. Neuro-Psiquiat., 9:368-371, 1951. 94. STEEGMANN, A. T. - Neuronitis of the cranial nerves. J. Nerv. a. Ment. Dis., 88:316-320, 1938. 95. STERNE, M. J. - Shyndrome de Guillain-Barré à type ascendant de Landry gravissime et curable: réaction de Paul Bunnel negative. Rev. Neurol., 80:786-787, 1948. 96. STILLMANN, S. J.; EANONG, W. F. - The Guillain-Barré syndrome; report of a case treated with ACTH and cortisone. New England J. Med., 246:293-296, 1952. 97. TAYLOR, E. W.; Mc DONALD, C. A. - The syndrome of polyneuritis with facial diplegia. Arch. Neurol. a. Psychiat., 27:79-112, 1932. 98. THOMAS, A.; PHELIPEAU, J. — Sur un syndrome complexe caractérisé par une paralysie atrophique facio-oculo-linguo-laryngo-vélopalatine, associée à une paralysie atrophique des membres inférieures, à l'abolition 
générale des reflexes tendineux, à tares multiples et la dissociation albumino-cytologique du liquide céphalo-rachidien. Rev. Neurol., 2:102-110, 1925. 99. THOMAS, A.; RENDU, H. - Sur un syndrome caractérisé par une diplegie faciale et des signes de polyneurite, hyperalbuminose du liquide céphalo-rachidien. Ses rapports possibles avec l'encéphalite épidemique. Rev. Neurol., 9:758-762, 1925. 100. TOLENTINO, P.; CRISALLI, M.; SCARABICCHI, S. - Considerazioni su cinque casi di meningopollradicolite (sindrome di Guillain-Barrẻ) trattati con la terapia ormonale. Minerva Med., 46:801-869, 1955. 101. TRABAUD, M. J. - Un nouveau cas de syndrome de Guillain-Barré d'origine spécifique. Rev. Neurol., 2:592-594, 1929.102. VAMPRE, E.; MINDLIN, H. S. - Polyradiculonevrites generalizadas: a propósito de 6 casos. Rev. Paulista de Med., 18:67-96, 1941. 103. VAN BOGARERT, L.; PHILLIPS, F.; RADERMECKER, J.; VERSCHRAEGEN, Th. - Les polyradiculonévrites avec dissociation albumino-cytologique et à évolution favorable. Essai sur un groupe épidémique de cas de polyradiculonévrite avec dissociation albumino-cytologique type de Guillain-Barré chez l'enfant et chez l'adulte. J. Belge de Neurol. et Psychiat., 38:151-211, 1938. 104. VAN BOGAERT, L.; MAERE, M. - Les polyradiculonévrites crâniennes bilaterales avec dissociation albumino-cytologique. Forme crânienne des polyradiculonévrites type Guillain-Barré. J. Belge de Neurol. et Psychiat., 38:275281, 1938. 105. VAN GEHUCHTEN, P. - Un cas de polyradiculonévrite avec dissociation albumino-cytologique et œè̀me de la papile. J. Belge de Neurol. et Psychiat., 38:212-216, 1938. 106. VON HAGEN, K. O.; BAKER, R. N. - Infectious neuronitis, present concepts of etiology and treatment. J.A.M.A., 151:1465-1472, 1953.107. WOLTMAN, H.; GOLDSTEIN, N. P. - Some where's and why's of neuritis. A consideration of structures and causes. Neurology, 8:187-192, 1958. 108. YOUNG, F. - Peripheral nerve paralyses following the use of various serums. Report of a case and review of the literature. J.A.M.A., 98:1139-1143, 1932. 1C9. ZIMMERMAN, H. J.; LOWRY, C. F. - Encephalomyeloradiculitis (Guillain-Barré syndrome) as complication of infectious hepatitis. Ann. Int. Med., 26:934-936, 1947.

Serviço de Neurologia da Escola Paulista de Medicina - Caixa Postal 5/96 -São Paulo, Brasil. 